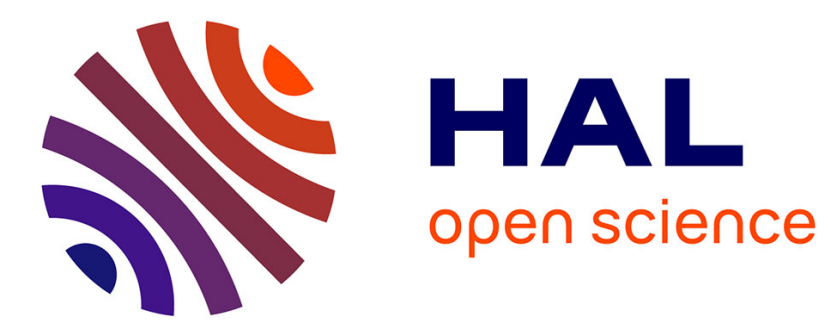

\title{
The effective permeability of fractured porous media subject to the Beavers-Joseph contact law
} Isabelle Gruais, Dan Polisevski, Florentina-Alina Stanescu

\section{To cite this version:}

Isabelle Gruais, Dan Polisevski, Florentina-Alina Stanescu. The effective permeability of fractured porous media subject to the Beavers-Joseph contact law. Asymptotic Analysis, 2014, 90 (3-4), pp.267280. 10.3233/ASY-141248 . hal-00904846

\section{HAL Id: hal-00904846}

\section{https://hal-univ-rennes1.archives-ouvertes.fr/hal-00904846}

Submitted on 15 Nov 2013

HAL is a multi-disciplinary open access archive for the deposit and dissemination of scientific research documents, whether they are published or not. The documents may come from teaching and research institutions in France or abroad, or from public or private research centers.
L'archive ouverte pluridisciplinaire HAL, est destinée au dépôt et à la diffusion de documents scientifiques de niveau recherche, publiés ou non, émanant des établissements d'enseignement et de recherche français ou étrangers, des laboratoires publics ou privés. 


\title{
The effective permeability of fractured porous media subject to the Beavers-Joseph contact law
}

\author{
Isabelle Gruais $^{\mathrm{a}}$, Dan Poliševski ${ }^{\mathrm{b}}$, Florentina-Alina Stanescu ${ }^{\mathrm{b}}$ \\ ${ }^{a}$ Université de Rennes 1, I.R.M.A.R, Campus de Beaulieu, 35042 Rennes Cedex (France) \\ ${ }^{b}$ I.M.A.R., P.O. Box 1-764, Bucharest (Romania).
}

\begin{abstract}
We are interested in the asymptotic behaviour of a fluid flow contained in a microscopic periodic distribution of fissures perturbating a porous medium where the Darcy law is valid, when the coupling between both systems is modeled by the Beavers-Joseph interface condition. As the small period of the distribution tends to zero, the interface condition is preserved on a microscopic scale under the additional assumption that the permeability coefficients behave like the squared period of the distribution which is also the squared size of the fissures. Moreover, the resulting pressure is purely macroscopic unlike the velocity field which also depends on the microscopic variable.
\end{abstract}

Keywords: Fractured porous media, Stokes flow, Beavers-Joseph interface, Homogenization, Two-scale convergence.

2000 MSC: 35B27, 76M50, 76S05, 76T99

\section{Introduction}

The perturbation of a porous medium by fissures containing a Stokes flow give rise to nontrivial phenomena that cannot be modeled by the laws of fluid mechanics. Given a microscopic periodically distributed collection of thin fissures, we are interested in the asymptotic behaviour of a medium where the Stokes equations of a fluid constrained in the fissures are coupled with the Darcy equations of the surrounding porous medium through a contact law of Beavers-Joseph type [6] known as the Saffman's variant [25]. The pioneering work by Beavers and Joseph yields the existence of a slip velocity at the interface between the fluid part and the fluid-saturated porous solid part. Proper rescaling of the fissures shows that the Beavers-Joseph condition 
influences the asymptotic behaviour of the system as long as the permeability coefficients obey one of two admissible alternatives. As one of them was studied in [10], we concentrate on the second alternative, namely the case where permeability is of the same order of magnitude as the squared period of the distribution. Our aim is is to show that although this influence is very small in comparison with the previous case it still plays a nongligeable part that could not be simply deduced from the first one.

As we use arguments of the homogenization theory, we briefly recall basic facts about the homogenization of fluids.

An argument favoring the use of periodic homogenization in fluid flows is that it was already used to justify Darcy's law [28]. Therefore, the present framework may be seen as a further development of this theory, although it does not deal with the formal method of asymptotic expansions [14], [15], [26] but rather extends the first rigorous proof based on the construction of a pressure extension due to [28] and followed by contextual variants [1], [16], [19]. with the restriction that, unlike previous works [1], [12], [28] relying on specific constructions, the velocity and pressure of the fluid have natural bounded extensions in the porous medium. We refer to [12] and references therein for developments about the physics and mathematics of this subject. However unrealistic, the periodicity assumption allows to concentrate ideas on the actual process. Homogenization of phenomena in fractured media were studied later in [22], [1] and [23] that is, when an assumption of nonconectedness could be dropped. Several models of fluid flows through fractured porous media (see [4], [5], [27], [9] and [24]) have been obtained by means of asymptotic methodswhere a homogeneous porous medium is altered by a possibly periodic distribution of microscopic fissures. Then, $\varepsilon$-periodicity allows to use procedures of the homogenization theory.

We consider an incompressible viscous fluid flow in a fractured porous media represented by a periodically structured domain consisting of two interwoven regions, separated by an interface. The first region represents the system of fissures which form the fracture, which is connected and where the flow is governed by the Stokes system. The second region, which is also connected, stands for the system of porous blocks, which have a certain permeability and where the flow is governed by Darcy's law. These two flows are coupled on the interface by the Saffman's variant [25] of the Beavers-Joseph condition [6], [17] which was confirmed by [13] as the limit of a homogenization process. Besides the continuity of the normal component of the velocity, it imposes the proportionality of the tangential velocity with the tangen- 
tial component of the viscous stress on the fluid-side of the interface. We prove here the existence and uniqueness of the solution of this model in our $\varepsilon$-periodic structure.

The paper is organized as follows. The problem of the flow is introduced in Section 2. More precisely, considering a microscopic periodic distribution of fissures we are interested in the behaviour of the equivalent material when the size of the fissures is of the same order of magnitude as the vanishing period of the distribution. The porous medium is described by a Darcy model in the solid part of the system and is coupled with Stokes equations in the fissures through a law of Beavers-Joseph type, see [6]. An adequate scaling shows that two cases arise depending on whether the permeability coefficients is of unity order or behave like the squared period of the distribution. Letting aside the first alternative which was studied in [10], we concentrate on the second one which displays new a priori estimates. The homogenization process is initiated in Section 3 thanks to a priori estimates and compacity arguments of the two-scale convergence theory (see [3], [18] and [21]) to identify convergent subsequences.

\section{The flow through the $\varepsilon$-periodic structure}

Let $\Omega$ be an open connected bounded set in $\mathbb{R}^{N}(N \geq 2)$, locally located on one side of the boundary $\partial \Omega$, a Lipschitz manifold composed of a finite number of connected components.

Let $Y_{f}$ be a Lipschitz open connected subset of the unit cube $\left.Y=\right] 0,1\left[{ }^{N}\right.$, such that the intersections of $\partial Y_{f}$ with $\partial Y$ are reproduced identically on the opposite faces of the cube and $0 \notin \bar{Y}_{f}$. The outward normal on $\partial Y_{f}$ is denoted by $\nu$. Repeating $Y$ by periodicity, we assume that the reunion of all the $\bar{Y}_{f}$ parts, denoted by $\mathbb{R}_{f}^{N}$, is a connected domain in $\mathbb{R}^{N}$ with a boundary of class $\mathcal{C}^{2}$. Defining $Y_{s}=Y \backslash \bar{Y}_{f}$, we assume also that the reunion of all the $\bar{Y}_{s}$ parts is a connected domain in $\mathbb{R}^{N}$.

For any $\varepsilon \in] 0,1[$ we denote

$$
\begin{gathered}
Z_{\varepsilon}=\left\{k \in \mathbb{Z}^{N}, \varepsilon k+\varepsilon Y \subseteq \Omega\right\} \\
I_{\varepsilon}=\left\{k \in Z_{\varepsilon}, \varepsilon k \pm \varepsilon e_{i}+\varepsilon Y \subseteq \Omega, \forall i \in \overline{1, N}\right\}
\end{gathered}
$$

where $e_{i}$ are the unit vectors of the canonical basis in $\mathbb{R}^{N}$.

Finally, we define the system of fissures by

$$
\Omega_{\varepsilon f}=\operatorname{int}\left(\cup_{k \in I_{\varepsilon}}\left(\varepsilon k+\varepsilon \bar{Y}_{f}\right)\right)
$$


and the porous matrix of our structure by $\Omega_{\varepsilon s}=\Omega \backslash \bar{\Omega}_{\varepsilon f}$. The interface between the porous blocks and the fluid is denoted by $\Gamma_{\varepsilon}=\partial \Omega_{\varepsilon f}$. Its normal is:

$$
\nu^{\varepsilon}(x)=\nu\left(\frac{x}{\varepsilon}\right), \quad x \in \Gamma_{\varepsilon}
$$

where $\nu$ has been periodically extended to $\mathbb{R}^{N}$.

Let us remark that $\Omega_{\varepsilon s}$ and $\Omega_{\varepsilon f}$ are connected and that the fracture ratio of this structure is given by

$$
\left.m=\left|Y_{f}\right| \in\right] 0,1\left[, \quad \text { as } \quad \frac{\left|\Omega_{\varepsilon f}\right|}{|\Omega|} \rightarrow m \quad \text { when } \quad \varepsilon \rightarrow 0 .\right.
$$

To the previous structure we associate a model of fluid flow through a fractured porous medium by assuming that there is a filtration flow in $\Omega_{\varepsilon s}$ obeying the Darcy's law and that there is a viscous flow in $\Omega_{\varepsilon f}$ governed by the Stokes system. These two flows are coupled by a Saffman's variant [25] of the Beavers-Joseph condition [6], [17]. This system is completed by an impermeability condition on $\partial \Omega$ :

$$
\begin{gathered}
\operatorname{div} v^{\varepsilon s}=0 \text { in } \Omega_{\varepsilon s} \\
\mu_{\varepsilon} v^{\varepsilon s}=K^{\varepsilon}\left(g^{\varepsilon}-\nabla p^{\varepsilon s}\right) \quad \text { in } \Omega_{\varepsilon s}, \\
\operatorname{div} v^{\varepsilon f}=0 \quad \text { in } \Omega_{\varepsilon f}, \\
\sigma_{i j}^{\varepsilon}=-p^{\varepsilon f} \delta_{i j}+2 \mu_{\varepsilon} e_{i j}\left(v^{\varepsilon f}\right) \text { in } \Omega_{\varepsilon f} \\
-\frac{\partial}{\partial x_{j}} \sigma_{i j}^{\varepsilon}=g_{i}^{\varepsilon} \quad \text { in } \Omega_{\varepsilon f} \\
v^{\varepsilon s} \cdot \nu^{\varepsilon}=v^{\varepsilon f} \cdot \nu^{\varepsilon} \text { on } \Gamma_{\varepsilon}, \\
-p^{\varepsilon s} \nu_{i}^{\varepsilon}-\sigma_{i j}^{\varepsilon} \nu_{j}^{\varepsilon}=\alpha_{\varepsilon} \mu_{\varepsilon} \beta_{\varepsilon}^{-1}\left(v_{i}^{\varepsilon f}-\left(v^{\varepsilon f} \cdot \nu^{\varepsilon}\right) \nu_{i}^{\varepsilon}\right) \quad \text { on } \Gamma_{\varepsilon}, \\
v^{\varepsilon s} \cdot n=0 \text { on } \partial \Omega, \quad n \text { the outward normal on } \partial \Omega
\end{gathered}
$$

where $v^{\varepsilon s}, v^{\varepsilon f}$ and $p^{\varepsilon s}, p^{\varepsilon f}$ stand for the corresponding velocities and pressures, $\mu^{\varepsilon}>0$ is the viscosity of the fluid, $\alpha_{\varepsilon} \in L^{\infty}(\Omega)$ is the positive nondimensional Beavers-Joseph number, $g^{\varepsilon} \in L^{2}(\Omega)^{N}$ is the exterior force and $e(v)$ denotes the symmetric tensor of the velocity gradient defined by

$$
e_{i j}(v)=\frac{1}{2}\left(\frac{\partial v_{i}}{\partial x_{j}}+\frac{\partial v_{j}}{\partial x_{i}}\right) \text {. }
$$


Finally, the positive tensor of permeability is defined by:

$$
K^{\varepsilon}(x)=\beta_{\varepsilon}^{2} K\left(\frac{x}{\varepsilon}\right),
$$

where $K \in L^{\infty}(Y)^{N \times N}$ and $\beta_{\varepsilon}>0$ stands for the magnitude of $\left(\operatorname{Tr} K^{\varepsilon}\right)^{1 / 2}$ with respect to $\varepsilon \rightarrow 0$.

As usual, we use the notations:

$$
\begin{gathered}
H_{0}(\operatorname{div}, \Omega)=\{v \in H(\operatorname{div}, \Omega), \quad v \cdot \nu=0 \quad \text { on } \quad \partial \Omega\} \\
L_{0}^{2}(\Omega)=\left\{p \in L^{2}(\Omega), \quad \int_{\Omega} p=0\right\} \\
V_{0}(\operatorname{div}, \Omega)=\left\{v \in H_{0}(\operatorname{div}, \Omega), \quad \operatorname{div} v=0 \quad \text { in } \quad \Omega\right\}
\end{gathered}
$$

Next, we define

$$
H_{\varepsilon}=\left\{v \in H_{0}(\operatorname{div}, \Omega), \quad v \in H^{1}\left(\Omega_{\varepsilon f}\right)^{N}\right\}
$$

the Hilbert space endowed with the scalar product

$$
(u, v)_{H_{\varepsilon}}=\int_{\Omega_{\varepsilon s}} u v+\int_{\Omega_{\varepsilon s}} \operatorname{div} u \operatorname{div} v+\varepsilon^{2} \int_{\Omega_{\varepsilon f}} e(u) e(v)+\varepsilon \int_{\Gamma_{\varepsilon}}\left(\gamma^{\varepsilon} u-\left(\gamma_{\nu}^{\varepsilon} u\right) \nu^{\varepsilon}\right) \gamma^{\varepsilon} v
$$

where $\gamma^{\varepsilon}$ and $\gamma_{\nu}^{\varepsilon}$ denote respectively the trace and the normal trace operators on $\Gamma_{\varepsilon}$ with respect to $\Omega_{\varepsilon f}$. Its corresponding subspace of incompressible velocities is

$$
V_{\varepsilon}=\left\{v \in V_{0}(\operatorname{div}, \Omega), \quad v \in H^{1}\left(\Omega_{\varepsilon f}\right)^{N}\right\}
$$

A useful property of the present structure is the existence of a bounded extension operator similar to that introduced in [7], [8] and [2] in the case of isolated fractures.

Theorem 2.1. There exists an extension operator $P_{\varepsilon}: H^{1}\left(\Omega_{\varepsilon f}\right) \rightarrow H_{0}^{1}(\Omega)$ such that

$$
\begin{gathered}
P_{\varepsilon} u=u \quad \text { in } \Omega_{\varepsilon f} \\
\left|e\left(P_{\varepsilon} u\right)\right|_{L^{2}(\Omega)} \leq C|e(u)|_{L^{2}\left(\Omega_{\varepsilon f}\right)}, \quad \forall u \in H^{1}\left(\Omega_{\varepsilon f}\right)
\end{gathered}
$$

where $C$ is independent of $\varepsilon$.

A straightforward consequence, via the corresponding Korn inequality, is 
Lemma 2.2. There exists some constant $C>0$, independent of $\varepsilon$, such that

$$
\left|u^{\varepsilon}\right|_{L^{2}\left(\Omega_{\varepsilon f}\right)}+\varepsilon|\nabla u|_{L^{2}\left(\Omega_{\varepsilon f}\right)} \leq C|u|_{H_{\varepsilon}}, \quad \forall u \in H_{\varepsilon} .
$$

Proof. We prove that:

$$
\left|e_{y}(u)\right|_{Y_{f}}^{2}+\left|\gamma u-\left(\gamma_{\nu} u\right) \nu\right|_{\Gamma}^{2}
$$

is a norm on $H^{1}\left(Y_{f}\right)$. Indeed, let

$$
\left|e_{y}\left(u^{k}\right)\right|_{Y_{f}}^{2}+\left|\gamma u^{k}-\left(\gamma_{\nu} u^{k}\right) \nu\right|_{\Gamma}^{2} \rightarrow 0
$$

and

$$
\left|u^{k}\right|_{Y_{f}}^{2}+\left|\nabla_{y} u^{k}\right|_{Y_{f}}^{2}=1
$$

From (2.26), we deduce that, at least for some subsequence and for some $u \in H^{1}\left(Y_{f}\right)$ :

$$
u^{k} \rightarrow u \quad \text { in } H^{1}\left(Y_{f}\right) .
$$

Moreover, (2.25) implies that

$$
e_{y}(u)=0 \text { and } \quad \gamma u-\left(\gamma_{\nu} u\right) \nu=0 .
$$

It follows that $u$ is a rigid displacement with vanishing the tangential trace on our $\Gamma$, that is $u \equiv 0$. The compactness of the inclusion $L^{2}\left(Y_{f}\right) \subset H^{1}\left(Y_{f}\right)$ leads to the strong convergence

$$
u^{k} \rightarrow u \quad \text { in } \quad L^{2}\left(Y_{f}\right) .
$$

Applying (2.26) again and Korn's inequality, we infer that (2.27) is a strong convergence, which leads to a contradiction between (2.26) and $u=0$. Finally, (2.23) is obtained by rescaling the fact that (2.24) is a norm on $H^{1}\left(Y_{f}\right)$.

\section{Denoting}

$$
A^{\varepsilon}=\left(K^{\varepsilon}\right)^{-1},
$$

and using the positivity of $K^{\varepsilon}$, we can assume without loss of genenerality that

$$
\exists a_{0}>0 \quad \text { such that } A_{i j}^{\varepsilon}(\cdot) \xi_{i} \xi_{j} \geq a_{0}|\xi|^{2}, \quad \forall \xi \in \mathbb{R}^{N}, \quad \text { a.e. in } \Omega .
$$

Rescaling the velocity by

$$
u^{\varepsilon}=\left\{\begin{array}{ll}
u^{\varepsilon s} & \text { in } \Omega_{\varepsilon s} \\
u^{\varepsilon f} & \text { in } \Omega_{\varepsilon f}
\end{array}=\frac{\mu_{\varepsilon}}{\beta_{\varepsilon}^{2}}\left\{\begin{array}{lll}
v^{\varepsilon s} & \text { in } & \Omega_{\varepsilon s} \\
v^{\varepsilon f} & \text { in } & \Omega_{\varepsilon f}
\end{array}\right.\right.
$$


then, for any $u, v \in H_{\varepsilon}$ and $q \in L_{0}^{2}(\Omega)$, we define

$$
\begin{gathered}
a_{\varepsilon}(u, v)=\int_{\Omega_{\varepsilon s}} A^{\varepsilon} u v+\beta_{\varepsilon}^{2} \int_{\Omega_{\varepsilon f}} e(u) e(v)+\beta_{\varepsilon} \int_{\Gamma_{\varepsilon}} \alpha_{\varepsilon}\left(\gamma^{\varepsilon} u-\left(\gamma_{\nu}^{\varepsilon} u\right) \nu^{\varepsilon}\right) \gamma^{\varepsilon} v \\
b_{\varepsilon}(q, v)=-\int_{\Omega} q \operatorname{div} v .
\end{gathered}
$$

We see that if the pair $\left(u^{\varepsilon}, p^{\varepsilon}\right)$ is a smooth solution of the problem (2.6)(2.13), then it is also a solution of the following problem: To find $\left(u^{\varepsilon}, p^{\varepsilon}\right) \in$ $H_{\varepsilon} \times L_{0}^{2}(\Omega)$ such that

$$
\begin{gathered}
a_{\varepsilon}\left(u^{\varepsilon}, v\right)+b_{\varepsilon}\left(p^{\varepsilon}, v\right)=\int_{\Omega} g^{\varepsilon} v, \quad \forall v \in H_{\varepsilon} \\
b_{\varepsilon}\left(q, u^{\varepsilon}\right)=0, \quad \forall q \in L_{0}^{2}(\Omega)
\end{gathered}
$$

Theorem 2.3. There exists a unique pair $\left(u^{\varepsilon}, p^{\varepsilon}\right) \in H_{\varepsilon} \times L_{0}^{2}(\Omega)$ solution of (2.33)-(2.34).

Proof. As $H_{0}^{1}(\Omega)$ is obviously included in $H_{\varepsilon}$, the following inf-sup condition is easily satisfied by $b_{\varepsilon}$ :

$$
\exists C_{1}^{\varepsilon}>0 \quad \text { such that } \inf _{q \in L_{0}^{2}(\Omega)} \sup _{v \in H_{\varepsilon}} \frac{b_{\varepsilon}(q, v)}{|v|_{H_{\varepsilon}}|q|_{L_{0}^{2}(\Omega)}} \geq C_{1}^{\varepsilon} .
$$

The coercivity condition (2.29) implies that

$$
\exists C_{2}^{\varepsilon}>0 \quad \text { such that } a_{\varepsilon}(v, v) \geq C_{2}^{\varepsilon}|v|_{H_{\varepsilon}}^{2}, \quad \forall v \in H_{\varepsilon},
$$

that is the $V_{\varepsilon}$-ellipticity of $a_{\varepsilon}$. As we also have

$$
V_{\varepsilon}=\left\{v \in H_{\varepsilon}, \quad b_{\varepsilon}(q, v)=0, \quad \forall q \in L_{0}^{2}(\Omega)\right\},
$$

the proof is completed by Corollary 4.1, Ch. 1 of [11].

In the rest of the paper we shall study the asymptotic behaviour (when $\varepsilon \rightarrow 0)$ of $\left(u^{\varepsilon}, p^{\varepsilon}\right)$, the unique solution of (2.33)-(2.34).

As $A_{\varepsilon}$ defined by (2.28) is of $\varepsilon^{0}$-order, we assume that

$$
\begin{array}{r}
\exists A \in L_{\text {per }}^{\infty}(Y)^{N^{2}} \text { such that } A^{\varepsilon}(x)=A\left(\frac{x}{\varepsilon}\right), x \in \Omega \\
\exists g \in L^{2}(\Omega)^{N} \quad \text { such that } g^{\varepsilon} \rightarrow g \text { strongly in } L^{2}(\Omega) .
\end{array}
$$


Because $\left|\Gamma_{\varepsilon}\right|$ is of $\varepsilon^{-1}$-order, we expect that macroscopic effects of the Beavers-Joseph condition will appear only when $\alpha_{\varepsilon} \beta_{\varepsilon}$ is of $\varepsilon^{1}$-order (see [20]). Therefore, we shall work under the hypothesis:

$\exists \alpha \in C_{\text {per }}^{1}(Y)$ and $\alpha_{0}>0$ such that $\varepsilon^{-1} \beta_{\varepsilon} \alpha_{\varepsilon}(x)=\alpha\left(\frac{x}{\varepsilon}\right) \geq \alpha_{0}, x \in \Omega$.

Thus, for the study of the asymptotic behaviour it remains only the order of $\beta_{\varepsilon}$ to be taken into account.

In the sequel we shall study the case when $\beta_{\varepsilon}=O(\varepsilon)$. Without loss of generality we can consider from now on that

$$
\exists \beta>0 \quad \text { such that } \quad \beta_{\varepsilon}^{2}=\varepsilon^{2} \beta, \quad \forall \varepsilon>0 .
$$

\section{The homogenization process}

From now on, for any function $\varphi$ defined on $\Omega \times Y$ we shall use the notations

$$
\begin{aligned}
& \varphi^{h}=\left.\varphi\right|_{\Omega \times Y_{h}}, \quad \tilde{\varphi}^{h}=\frac{1}{\left|Y_{h}\right|} \int_{Y_{h}} \varphi(\cdot, y) d y, \quad h \in\{s, f\}, \\
& \tilde{\varphi}=\int_{Y} \varphi(\cdot, y) d y, \quad \text { that is } \tilde{\varphi}=(1-m) \tilde{\varphi}^{s}+m \tilde{\varphi}^{f} .
\end{aligned}
$$

Also, for any sequence $\left(\varphi^{\varepsilon}\right)_{\varepsilon}$, bounded in $L^{2}(\Omega \times Y)$, we denote

$$
\varphi^{\varepsilon} \stackrel{2}{\rightarrow} \varphi
$$

iff $\varphi^{\varepsilon}$ is two-scale convergent to $\varphi \in L^{2}(\Omega \times Y)$ in the sense of [3].

Noticing that $u^{\varepsilon} \in V_{\varepsilon}$ and setting $v=u^{\varepsilon}$ in (2.33) we get

$$
\left|u^{\varepsilon}\right|_{H_{\varepsilon}}^{2} \leq C\left|u^{\varepsilon}\right|_{L^{2}(\Omega)} \text {. }
$$

Applying (2.23) we find that

$$
\begin{gathered}
\left\{u^{\varepsilon}\right\}_{\varepsilon} \text { is bounded in } V_{\varepsilon} \text { and in } V_{0}(\operatorname{div}, \Omega), \\
\left|u^{\varepsilon}\right|_{L^{2}\left(\Omega_{\varepsilon f}\right)}+\varepsilon\left|\nabla u^{\varepsilon}\right|_{L^{2}\left(\Omega_{\varepsilon f}\right)} \leq C, \quad C \text { being independent of } \varepsilon .
\end{gathered}
$$

It follows that $\exists u \in L^{2}(\Omega \times Y)^{N}$ such that, on some subsequence

$$
u^{\varepsilon} \stackrel{2}{\rightarrow} u
$$




$$
u^{\varepsilon} \rightarrow \int_{Y} u(\cdot, y) d y \in V_{0}(\operatorname{div}, \Omega) \quad \text { weakly in } \quad L^{2}(\Omega)^{N}
$$

Denoting $\chi_{\varepsilon f}(x)=\chi_{f}\left(\frac{x}{\varepsilon}\right)$ and $\chi_{\varepsilon s}(x)=\chi_{s}\left(\frac{x}{\varepsilon}\right)$, where $\chi_{f}$ and $\chi_{s}$ are the characteristic functions of $Y_{f}$ and $Y_{s}$ in $Y$, we see that $\left(\chi_{\varepsilon s} u^{\varepsilon}\right)_{\varepsilon},\left(\chi_{\varepsilon f} u^{\varepsilon}\right)_{\varepsilon}$ and $\left(\chi_{\varepsilon f} \frac{\partial u^{\varepsilon}}{\partial x_{i}}\right)_{\varepsilon}$ are bounded in $\left(L^{2}(\Omega)\right)^{N}, \forall i \in\{1,2, \cdots, N\}$.

Let us denote:

$$
\tilde{H}_{\mathrm{per}}^{1}\left(Y_{f}\right)=\left\{\varphi \in H_{\mathrm{loc}}^{1}\left(\mathbb{R}_{f}^{N}\right), \quad \varphi \quad \text { is } Y \text {-periodic, } \quad \int_{Y_{f}} \varphi=0\right\} .
$$

Lemma 3.1. $u^{f} \in L^{2}\left(\Omega,\left(\tilde{H}_{\mathrm{per}}^{1}\left(Y_{f}\right)\right)^{N}\right)$ and satisfies:

$$
\varepsilon \chi_{\varepsilon f} \nabla u_{i}^{\varepsilon} \stackrel{2}{\rightarrow} \chi_{f} \nabla_{y} u_{i}^{f}
$$

Proof. Using the compacity result of [21], it follows that $\exists \eta_{i} \in L^{2}(\Omega \times Y)^{N}$ such that, on some subsequence we have

$$
\varepsilon \chi_{\varepsilon f} \nabla u_{i}^{\varepsilon} \stackrel{2}{\rightarrow} \eta_{i}
$$

Next, we easily remark that $\left.\eta_{i}\right|_{\Omega \times Y_{s}}=0$.

Let us introduce

$$
\begin{gathered}
V_{0}^{\text {per }}\left(\operatorname{div}, Y_{f}\right)=\left\{\varphi \in H_{\text {loc }}\left(\operatorname{div}, \mathbb{R}_{f}^{N}\right), \operatorname{div}_{y} \varphi=0 \text { in } \mathbb{R}_{f}^{N},\right. \\
\varphi \cdot \nu=0 \text { on } \Gamma, \varphi \text { is } Y-\text { periodic }\} .
\end{gathered}
$$

Let $\psi \in L^{2}\left(\Omega, V_{0}^{\text {per }}\left(\operatorname{div}, Y_{f}\right)\right)$; denoting $\psi^{\varepsilon}=\psi\left(x, \frac{x}{\varepsilon}\right)$, we have

$$
\begin{gathered}
\psi^{\varepsilon} \in H^{1}\left(\Omega_{\varepsilon f}\right)^{N} \text { and } \psi^{\varepsilon} \nu^{\varepsilon}=0 \text { on } \Gamma_{\varepsilon} \\
\int_{\Omega_{\varepsilon f}} \varepsilon \nabla u_{i}^{\varepsilon}(x) \psi^{\varepsilon}(x) d x=-\int_{\Omega_{\varepsilon f}} \varepsilon u_{i}^{\varepsilon}(x) \operatorname{div}_{x} \psi\left(x, \frac{x}{\varepsilon}\right) d x
\end{gathered}
$$

Using the two-scale convergences (3.6)-(3.10) we find

$$
\int_{\Omega \times Y_{f}} \eta_{i}(x, y) \psi(x, y) d x d y=0, \quad \psi \in L^{2}\left(\Omega, V_{0}^{p e r}\left(\operatorname{div}, Y_{f}\right)\right) .
$$

As the orthogonal space of $V_{0}^{\text {per }}\left(\operatorname{div}, Y_{f}\right)$ in $L^{2}(\Omega \times Y)$ is:

$$
\nabla \tilde{H}_{p e r}^{1}\left(Y_{f}\right)=\left\{\nabla q, \quad q \in \tilde{H}_{p e r}^{1}\left(Y_{f}\right)\right\}
$$


(see Th.2.7, Ch.I[11]), it follows that there exists $w \in L^{2}\left(\Omega ; \tilde{H}_{p e r}^{1}\left(Y_{f}\right)^{N}\right)$ such that

$$
\eta_{i}(x, y)=\chi_{f}(y) \nabla_{y} w_{i}(x, y), \quad \forall i \in\{1, \ldots, N\} .
$$

Further we prove that

$$
w_{i}(x, y)=u_{i}^{f}(x, y)-\tilde{u}_{i}^{f}(x), \quad \forall i \in\{1, \ldots, N\},
$$

which complets the proof.

Proposition 3.2. There holds:

$$
\begin{gathered}
\operatorname{div}_{y} u^{f}=0, \quad \text { in } \Omega \times Y_{f} \\
\operatorname{div}_{y} u^{s}=0, \quad \text { in } \Omega \times Y_{s} \\
u_{n}^{f}=u_{n}^{s}, \quad \text { on } \Omega \times \Gamma \\
(1-m) \operatorname{div} \tilde{u}^{s}+m \operatorname{div} \tilde{u}^{f}=0, \quad \text { in } \Omega .
\end{gathered}
$$

Proof. First, let $a \in\{s, f\}$ and let $\zeta \in \mathcal{D}(\Omega), \theta \in \mathcal{D}\left(\Omega \times Y_{a}\right)$. Define

$$
\theta^{\varepsilon}=\theta\left(x, \frac{x}{\varepsilon}\right) \text {. }
$$

There holds

$$
\begin{gathered}
0=\int_{\Omega_{\varepsilon_{s}}} \operatorname{div} u^{\varepsilon}\left(\zeta+\varepsilon \theta^{\varepsilon}\right)+\int_{\Omega_{\varepsilon f}} \operatorname{div} u^{\varepsilon}\left(\zeta+\varepsilon \theta^{\varepsilon}\right)= \\
=-\int_{\Omega_{\varepsilon_{s}}} u^{\varepsilon}\left(\nabla \zeta+\varepsilon(\nabla \theta)^{\varepsilon}+\nabla_{y} \theta^{\varepsilon}\right)-\int_{\Omega_{\varepsilon f}} u^{\varepsilon}\left(\nabla \zeta+\varepsilon(\nabla \theta)^{\varepsilon}+\nabla_{y} \theta^{\varepsilon}\right) \\
\rightarrow-\int_{\Omega \times Y_{f}} u^{f}\left(\nabla \zeta+\nabla_{y} \theta\right)-\int_{\Omega \times Y_{s}} u^{s}\left(\nabla \zeta+\nabla_{y} \theta\right)= \\
=\int_{\Omega \times Y_{f}} \operatorname{div}_{y} u^{f} \theta+\int_{\Omega \times Y_{s}} \operatorname{div}_{y} u^{s} \theta+\int_{\Omega}\left(m \operatorname{div} \tilde{u}^{f}+(1-m) \operatorname{div} \tilde{u}^{s}\right) \zeta
\end{gathered}
$$

from which we deduce (3.17), (3.18) and (3.20).

Second, let $\zeta \in \mathcal{D}(\Omega), \theta \in \mathcal{D}\left(\Omega ; C_{\text {per }}^{\infty}(Y)\right)$ and define

$$
\theta^{\varepsilon}=\theta\left(x, \frac{x}{\varepsilon}\right) \text {. }
$$

There holds

$$
0=\int_{\Omega} \operatorname{div} u^{\varepsilon}\left(\zeta+\varepsilon \theta^{\varepsilon}\right)=-\int_{\Omega} u^{\varepsilon}\left(\nabla \zeta+\varepsilon(\nabla \theta)^{\varepsilon}+\nabla_{y} \theta^{\varepsilon}\right)+\int_{\Gamma_{\varepsilon}}\left[u_{n}^{\varepsilon}\right]\left(\zeta+\varepsilon \theta^{\varepsilon}\right)=
$$




$$
\begin{gathered}
=-\int_{\Omega} u^{\varepsilon}\left(\nabla \zeta+\varepsilon(\nabla \theta)^{\varepsilon}+\nabla_{y} \theta^{\varepsilon}\right) \rightarrow-\int_{\Omega \times Y_{f}} u^{f}\left(\nabla \zeta+\nabla_{y} \theta\right)-\int_{\Omega \times Y_{s}} u^{s}\left(\nabla \zeta+\nabla_{y} \theta\right)= \\
=\int_{\Omega \times Y_{f}} \operatorname{div}_{y} u^{f} \theta+\int_{\Omega \times Y_{s}} \operatorname{div}_{y} u^{s} \theta+|Y| \int_{\Omega}\left(\operatorname{div} \tilde{u}^{f}+\operatorname{div} \tilde{u}^{s}\right) \zeta-\int_{\Omega \times \Gamma}\left(u_{n}^{f}-u_{n}^{s}\right) \theta \\
=-\int_{\Omega \times \Gamma}\left(u_{n}^{f}-u_{n}^{s}\right) \theta
\end{gathered}
$$

which yields (3.19) and achieves the proof.

The same arguments as in [10] yield:

Proposition 3.3. There exists a constant $C>0$ independent of $\varepsilon$ such that

$$
\left|p^{\varepsilon}\right|_{L^{2}(\Omega)}+\left|\nabla p^{\varepsilon}\right|_{L^{2}\left(\Omega_{\varepsilon s}\right)} \leq C
$$

Lemma 3.4. There exists $p \in L_{0}^{2}(\Omega \times Y)$ with $p^{s}=\tilde{p}^{s} \in H^{1}(\Omega)$ and $p^{f}=$ $\tilde{p}^{f} \in L^{2}(\Omega)$ such that, up to some subsequence, we have:

$$
p^{\varepsilon} \stackrel{2}{\rightarrow} p
$$

Moreover: $p^{s}=p^{f}=p$ and thus $p \in \tilde{H}^{1}(\Omega)$.

Proof. The same arguments as in [10] yield $p^{s}=\tilde{p}^{s} \in L^{2}(\Omega)$. Let

$$
Q_{\varepsilon}: H^{1}\left(\Omega_{\varepsilon s}\right) \rightarrow H^{1}(\Omega)
$$

denote the continuous extension operator introduced in [7]. From (3.21), we deduce that, at least for some subsequence, $\exists z \in H^{1}(\Omega)$ such that

$$
Q_{\varepsilon} p^{\varepsilon} \rightarrow z \quad \text { in } H^{1}(\Omega) .
$$

Noticing that $\chi_{\varepsilon s} Q_{\varepsilon} p^{\varepsilon}=\chi_{\varepsilon s} p^{\varepsilon}$ in $\Omega$, we deduce that that $z=\tilde{p}^{s}$ in $H^{1}(\Omega)$, which yields

$$
p^{s}=\tilde{p}^{s} \in H^{1}(\Omega) .
$$

Using (2.9)-(2.10) and noticing that Murat-Tartar inequality together with Korn's inequality yield, for some constant $C>0$ independent of $\varepsilon$ :

$$
|v|_{L^{2}\left(\Omega_{\varepsilon f}\right)} \leq C|e(v)|_{L^{2}\left(\Omega_{\varepsilon f}\right)}, \quad \forall v \in H_{0}^{1}\left(\Omega_{\varepsilon f}\right)
$$

we find that there exists a constant $C>0$ such that

$$
\left|\nabla p^{\varepsilon}\right|_{H^{-1}\left(\Omega_{\varepsilon f}\right)} \leq C \varepsilon \text {. }
$$


Theorem 3.2 of [23] yields the existence of $p^{f} \in L^{2}(\Omega)$ such that, on some subsequence:

$$
\chi_{\varepsilon f} p^{\varepsilon} \stackrel{2}{\rightarrow} \chi_{f} p^{f}
$$

To conclude, let $\varphi \in \mathcal{D}(\Omega), \psi \in \mathcal{C}_{\text {per }}^{\infty}(Y)^{N}$ such that

$$
\int_{\Gamma} \psi \cdot \nu \neq 0
$$

and set

$$
v_{i}^{\varepsilon}(x)=\varepsilon \varphi(x) \psi_{i}\left(\frac{x}{\varepsilon}\right), \quad \forall i \in\{1, \cdots, N\} .
$$

Using $v^{\varepsilon}$ in the variational formulation (2.33)-(2.34) and passing to the limit as $\varepsilon \rightarrow 0$ yields

$$
\int_{\Omega}\left(p^{f}(x)-p^{s}(x)\right) \varphi(x) d x\left(\int_{\Gamma} \psi \cdot \nu\right)=0, \quad \forall \varphi \in \mathcal{D}(\Omega)
$$

that is, $p^{s}=p^{f}$. We conclude thanks to $(3.23)$.

\section{The homogenized problem}

Consider the Hilbert space:

$X=\left\{u \in L^{2}(\Omega \times Y), u^{f} \in L^{2}\left(\Omega, \tilde{H}_{\mathrm{per}}^{1}\left(Y_{f}\right)\right),\left(\tilde{u}^{s}, \tilde{u}^{f}\right) \in H_{0}(\operatorname{div}, \Omega)^{2}, \operatorname{div}_{y} u=0\right\}$

endowed with the scalar product:

$(u, v)_{X}=\int_{\Omega \times Y_{s}} u v+\int_{\Omega} \operatorname{div} \tilde{u} \operatorname{div} \tilde{v}+\int_{\Omega \times Y_{f}} e_{y}(u) e_{y}(v)+\int_{\Omega \times \Gamma} \alpha(y)\left(\gamma u \gamma v-\gamma_{\nu} u \gamma_{\nu} v\right)$

and set:

$$
X_{0}=\{u \in X, \operatorname{div} \tilde{u}=0\}, \quad M=L_{0}^{2}(\Omega) .
$$

We can present our first homogenization result:

Proposition 4.1. The limit problem reads: Find $(v, q) \in X \times M$ such that

$$
\begin{gathered}
a(v, \varphi)+b(q, \varphi)=\int_{\Omega} g \tilde{\varphi}, \quad \forall \varphi \in X \\
b(\pi, v)=0, \quad \forall \pi \in M
\end{gathered}
$$


where we set

$$
\begin{gathered}
a(v, \varphi)=\int_{\Omega \times Y_{s}} A v \varphi+\beta \int_{\Omega \times Y_{f}} e_{y}(v): e_{y}(\varphi)+\int_{\Omega \times \Gamma} \alpha(y)\left(\gamma v-\left(\gamma_{\nu} v\right) \nu\right) \gamma \varphi \\
b(\pi, v)=-\int_{\Omega} \pi \operatorname{div} \tilde{v},
\end{gathered}
$$

Proof. Let $\varphi \in X \cap \mathcal{C}^{\infty}(\Omega \times Y)$ and set

$$
\varphi^{\varepsilon}(x)=\varphi\left(x, \frac{x}{\varepsilon}\right) .
$$

Then $\varphi^{\varepsilon} \in V_{\varepsilon}$. Passing to the limit in (2.33) with $\varphi^{\varepsilon}$ as the test function, we obtain (4.1). Notice that the integral on $\Omega \times \Gamma$ results from the same arguments as in [10].

Let $\pi \in M$ and set likewise:

$$
\pi^{\varepsilon}(x)=\pi\left(x, \frac{x}{\varepsilon}\right) .
$$

Then, $\pi^{\varepsilon} \in L_{0}^{2}(\Omega)$ and we may pass to the limit in (2.34) with $\pi^{\varepsilon}$ as the test function to obtain (4.2).

Proposition 4.2. The problem (4.1)-(4.2) is well-posed.

Proof. Let $\pi \in M$. Using Theorem 4.2 of [10], let $v \in H_{0}^{1}(\Omega)^{N} \subset X$ be the unique solution of $\pi=\operatorname{div} v$ such that $|v|_{H_{0}^{1}(\Omega)} \leq C|\pi|_{L^{2}(\Omega)}$.

Noticing that

$$
|v|_{X}^{2} \leq C|v|_{H^{1}(\Omega)}^{2} \leq C|\pi|_{L^{2}(\Omega)}^{2}
$$

we deduce that:

$$
\sup _{\varphi \in X} \frac{b(\pi, \varphi)}{|\varphi|_{X}|\pi|_{\Omega}} \geq \frac{b(\pi, v)}{|v|_{X}|\pi|_{\Omega}}=\frac{|\pi|_{\Omega}^{2}}{|v|_{X}|\pi|_{\Omega}} \geq \frac{1}{C}
$$

The coercivity of $a(\cdot, \cdot)$ follows from the coercivity of the matrix $A$ and that $X$ may be equipped with the norm (2.24). We conclude as in Theorem 4.2 of [10] using Corollary 4.1, Ch. 1 of [11].

Proposition 4.3. The problem (4.1)-(4.2) equivalently reads: Find $u \in X_{0}$ such that

$$
a(u, \varphi)=\int_{\Omega}(g-\nabla p) \tilde{\varphi}, \quad \forall \varphi \in X_{0}
$$


Consider the cell problem: for every $i \in\{1, \cdots, N\}$, let $w^{i} \in W$, where:

$$
W=\left\{w \in L^{2}(Y), \quad u^{f} \in \tilde{H}_{\mathrm{per}}^{1}\left(Y_{f}\right), \quad \operatorname{div}_{y} w=0 \quad \text { in } \quad Y\right\}
$$

such that

$$
\int_{Y_{s}} A w^{i} \psi+\beta \int_{Y_{f}} e_{y}\left(w^{i}\right): e_{y}(\psi)+\int_{\Gamma} \alpha\left(\gamma w^{i}-\left(\gamma_{\nu} w^{i}\right) \nu\right) \psi=\int_{Y} \psi_{i}, \quad \forall \psi \in W .
$$

Then, using the convention of repeated indices, the solution of the homogenized problem reads:

$$
u(x, y)=w^{i}(y)\left(g_{i}(x)-\frac{\partial p}{\partial x_{i}}\right)
$$

We set

$$
K_{j i}=\int_{Y} w_{j}^{i}
$$

Proposition 4.4. The effective permeability tensor $K$ is symmetric and positively defined.

Proof. By definition of $w^{i}$, the matrix $K$ is symmetric. Introducing $w^{\xi}=$ $\sum_{i=1}^{N} \xi_{i} w^{i}$ for every $\xi \in \mathbb{R}^{N}$, we see that $K$ is also positively definite. Therefore, the positivity is given by the lowest, necessarily positive, eigenvalue of $K$.

From (4.5) we get the Darcy's law

$$
\tilde{u}=K(g-\nabla p),
$$

where $\tilde{u} \in H_{0}(\operatorname{div}, \Omega)$ and $p \in \tilde{H}^{1}(\Omega)$ is the unique solution of the following boundary value problem:

$$
\begin{array}{r}
\operatorname{div}(K \nabla p)=\operatorname{div}(K g) \quad \text { in } \quad \Omega, \\
K \nabla p \cdot n=K g \cdot n \quad \text { on } \quad \partial \Omega,
\end{array}
$$

where obviously $\operatorname{div}(K g) \in H^{-1}(\Omega)$.

Proposition 4.5. The problem (4.8)-(4.9) is well-posed. 
Proof. As $K$ is symmetric and positively defined, (4.8)-(4.9) is a clasical non homogeneous Neumann problem. The compatibility condition is obviously satisfied. Then there exists a unique solution in $\tilde{H}^{1}(\Omega)$.

Acknowledgements. This work has been accomplished during the visit of Florentina-Alina Stanescu at the I.R.M.A.R's Department of Mechanics (University of Rennes 1); her contribution to this paper is supported by the Sectorial Operational Programme Human Resources Development (SOP HRD), financed from the European Social Fund and by the Romanian Government under the contract number SOP HRD/107/1.5/S/82514.

\section{References}

[1] G. Allaire, Homogenization of the Stokes flow in a connected porous medium, Asymptotic Analysis 2 (1989) 203-222.

[2] G. Allaire, Homogenization of the Navier-Stokes Equations with a Slip Boundary Condition, Communications in Pure and Applied Mathematics 44 (1991) 605-641.

[3] G. Allaire, Homogenization and two-scale convergence, S.I.A.M. J. Math. Anal. 23 (1992) 1482-151.

[4] G.I. Barenblatt, Y.P. Zheltov, I.N. Kochina, On basic conceptions of the theory of homogeneous fluids seepage in fractured rocks (in Russian), Prikl.Mat. i Mekh. 24 (1960) 852-864.

[5] G.I. Barenblatt, V.M. Entov, V.M. Ryzhik, Theory of Fluid Flows Through Natural Rocks, Kluwer Acad. Pub., Dordrecht, 1990.

[6] G.S. Beavers, D.D. Joseph, Boundary conditions at a naturally permeable wall, J. Fluid Mech. 30 (1967) 197-207.

[7] D. Cioranescu, J. Saint-Jean-Paulin, Homogenization in open sets with holes, J. Math. Anal. Appl. 71 (2)(1979) 590-607

[8] C. Conca, On the application of the homogenization theory to a class of problems arising in fluid mechanics, J. Math. Pures et Appl. 64(1985) $31-75$ 
[9] H.I. Ene, D. Poliševski, Model of diffusion in partially fissured media, Z.A.M.P. 53 (2002) 1052-1059.

[10] I. Gruais, D. Poliševski, Fluid flows through fractured porous media along Beavers-Joseph interfaces Preprint I.R.M.A.R. 2013.

[11] V. Girault, P.-A. Raviart Finite Element Methods for Navier-Stokes Equations, Springer-Verlag, Berlin, 1986.

[12] U. Hornung, Homogenization and porous media,Interdiscip. Appl. Math.6, Springer,New York(1997).

[13] W. Jäger, A. Mikelić, Modeling effective interface laws for transport phenomena between an unconfined fluid and a porous medium using homogenization, Transp. Porous Med. 78 (2009) 489-508.

[14] J.B. Keller, Darcy's law for flow in porous media and the two-space method, Nonlinear partial differential equations in engineering and applied science (Proc. Conf., Univ. Rhode Island, Kingston, R.I., 1979), Lecture Notes in Pure and Appl. Math. 54 429-443, Dekker,New $\operatorname{York}(1980)$

[15] J.-L Lions, Some methods in the mathematical analysis of systems and their control, Kexue Chubanshe (Science Press), Beijing(1981)

[16] R. Lipton and M. Avellaneda, Darcy's law for slow viscous flow past a stationary array of bubbles, Proc. Roy. Soc. Edinburgh Sect. A 114 $(1-2)(1990) 71-79$.

[17] I.P. Jones, Low Reynolds number flow past a porous spherical shell, Proc Camb. Phil. Soc. 73 (1973) 231-238.

[18] D. Lukkassen, G. Nguetseng, P. Wall, Two-scale convergence. Int. J. Pure Appl. Math. 2 (2002) 35-86.

[19] A. Mikelić, Effets inertiels pour un écoulement stationnaire visqueux incompressible dans un milieu poreux, C. R. Acad. Sci. Paris Sér. I Math.320 (10) (1995) 1289-1294.

[20] M. Neuss-Radu, Some extensions of two-scale convergence. C.R. Acad. Sci. Paris, t. 322, Serie I (1996) 899-904. 
[21] G. Nguetseng, A general convergence result for a functional related to the theory of homogenization, S.I.A.M. J.Math.Anal. 20 (1989) 608-623.

[22] D. Poliševski, On the homogenization of fluid flows through periodic media, Rend. Sem. Mat. Univers. Politecn. Torino 45 (2) (1987) 129-139.

[23] D. Poliševski, Basic homogenization results for a biconnected $\epsilon$-periodic structure, Appl. Anal. 82 (4) (2003) 301-309.

[24] D. Poliševski, The Regularized Diffusion in Partially Fractured Porous Media, in: L. Dragos (Ed.) Current Topics in Continuum Mechanics, Volume 2, Ed. Academiei, Bucharest, 2003.

[25] P.G. Saffman, On the boundary condition at the interface of a porous medium. Stud. Appl. Math. 50 (1971) 93-101.

[26] E. Sanchez-Palencia, Non-Homogeneous Media and Vibration Theory. Lecture Notes in Physics, Vol. 127, Springer-Verlag, Berlin, 1980.

[27] R.E. Showalter, N.J. Walkington, Micro-structure models of diffusion in fissured media, J. Math. Anal. Appl. 155 (1991) 1-20.

[28] L. Tartar, Incompressible fluid flow in a porous medium. Convergence of the homogenization process. Appendix of [26]. 